\title{
Inelastic light scattering from a Mott insulator
}

\author{
D. van Oosten, ${ }^{1,2}$ D. B. M. Dickerscheid, ${ }^{1,3}$ B. Farid, ${ }^{1}$ P. van der Straten, ${ }^{2}$ and H. T. C. Stoof $^{1}$ \\ ${ }^{1}$ Institute for Theoretical Physics, University of Utrecht, Leuvenlaan 4, 3584 CE Utrecht, The Netherlands \\ ${ }^{2}$ Debye Institute, University of Utrecht, Princetonplein 5, 3584 CC Utrecht, The Netherlands \\ ${ }^{3}$ Lorentz Institute, Leiden University, P.O. Box 9506, 2300 RA Leiden, The Netherlands
}

(Received 20 May 2004; published 1 February 2005)

\begin{abstract}
We propose to use Bragg spectroscopy to measure the excitation spectrum of the Mott-insulator state of an atomic Bose gas in an optical lattice. We calculate the structure factor of the Mott insulator taking into account both the self-energy corrections of the atoms and the corresponding dressing of the atom-photon interaction. We determine the scattering rate of photons in the stimulated Raman transition and show that by measuring this scattering rate in an experiment, in particular, the excitation gap of the Mott insulator can be determined.
\end{abstract}

DOI: 10.1103/PhysRevA.71.021601

PACS number(s): 03.75.Hh, 32.80.Pj, 39.25.+k, 67.40.-w

\section{INTRODUCTION}

A Bose-Einstein condensate in an optical lattice is a powerful tool to investigate strongly correlated Bose gases [1,2]. In particular, the experiment by Greiner et al. [3] has shown that it is possible to achieve a quantum phase transition from a superfluid to a Mott-insulating phase in this system. The latter phase transition was predicted to occur in the BoseHubbard model by Fisher et al. [4], and Jaksch et al. [5] were the first to make the crucial observation that the BoseHubbard model can be applied to bosonic atoms in an optical lattice. The mean-field phase diagram at zero temperature was calculated by several authors [6-8], however, the Bogoliubov-like theory for the excitations in both the superfluid and the Mott-insulator phases of the gas was developed more recently by van Oosten et al. [9].

An important advantage of using atoms in an optical lattice to study the Bose-Hubbard model, is that the system is free from disorder, which makes it possible to make very accurate predictions and measurements. A good example of such a high-precision measurement is Bragg spectroscopy. This technique has already been used to coherently split a Bose-Einstein condensate into two momentum components [10], to measure the excitation spectrum of a trapped BoseEinstein condensate [11], and to measure the light-shifted energy levels of an atom in an optical lattice [12]. Here we propose to use Bragg spectroscopy to measure the excitation spectrum of the Mott-insulator state. In particular, one can in this way determine the value of the particle-hole gap in the excitation spectrum and study the behavior of this gap as the system approaches the quantum critical point. Note that the excitation spectrum as obtained using Bragg spectroscopy does not yield what is generally referred to as the Mott gap, because this gap is associated with single-particle excitations. The value of the particle-hole gap can be obtained though and is a particularly interesting quantity in the study of quantum critical phenomena. In addition, this gap is also very important for the practical application of these systems to quantum information processing, since the gap determines the fidelity of the Mott state.

In a Bragg-spectroscopy experiment, two laser beams are used to make excitations in the system, as shown in Fig. 1(a). The two lasers both have a large detuning with respect to an optical transition in the atoms so that spontaneous emission is suppressed. However, the relative detuning can be very small. When an atom absorbs a photon from beam 2 and is stimulated to emit a photon into beam 1, the atom undergoes a change of momentum $\hbar \mathbf{q}=\hbar \mathbf{k}_{2}-\hbar \mathbf{k}_{1}$ and a change of energy $\hbar \omega=\hbar \omega_{2}-\hbar \omega_{1}$. In principle any optical transition could be used, but here we use the same transition that is employed to create the lattice potential. This means that the magnitude of the momentum is given by $\hbar q=2 \hbar k_{\mathrm{ph}} \sin (\theta / 2)$, where to a good approximation $\hbar k_{\mathrm{ph}}=2 \pi \hbar / \lambda$ is the photon momentum of both the lasers, $\lambda$ is equal to the wavelength of the lattice laser light, and $\theta$ is the angle between the two laser beams. By varying the angle between the two laser beams, any momentum between zero and $2 \hbar k_{\mathrm{ph}}$ can be transferred, and by
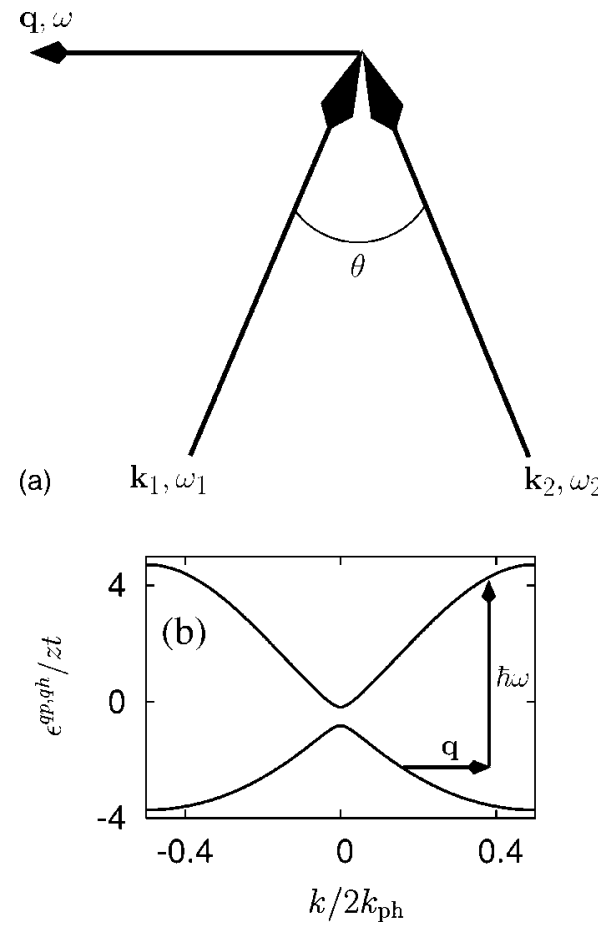

FIG. 1. (a) Setup for the proposed experiment. (b) Particle and hole dispersions in units of the tunneling parameter in a onedimensional lattice, for $U / z t=6$. The horizontal arrow indicates absorption of momentum, the vertical arrow absorption of energy. 
varying the relative detuning between the beams, the amount of energy that is transferred to the system can be controlled. Note that in experiments, there is always additional harmonic confinement present, which causes a shell of superfluid atoms around a Mott-insulating core [5]. This means that the momentum $\hbar q$ has to be chosen such that the corresponding wavelength is smaller than the size of the core.

Calculating the scattering rate for a given momentum $\hbar \mathbf{q}$ and energy $\hbar \omega$ involves counting the number of ways in which the requirements of momentum and energy conservation can be met. To illustrate this process, we draw in Fig. 1(b) the quasiparticle and quasihole dispersions in the Mott insulator [9], as is common in solid-state physics. The horizontal and vertical arrows in the figure indicate the transfer of momentum and energy, respectively. Since energy is deposited in the system, this scattering rate can be measured in a trap-loss experiment, or by determining the increase in temperature of the atoms.

\section{THE SCATTERING RATE}

To calculate the desired two-photon scattering rate we use Fermi's golden rule. This can be expressed as $I(\mathbf{q}, \omega)$ $=-2 \operatorname{Im}[\Pi(\mathbf{q}, \omega)] / \hbar$, where $\Pi(\mathbf{q}, \omega)$ is the polarizibility of the medium. The polarizibility can be written as $\Pi(\mathbf{q}, \omega)$ $=(\hbar \Omega / 2)^{2} \chi(\mathbf{q}, \omega)$, with $\Omega$ the effective Rabi frequency for the two-photon process and $\chi$ the susceptibility. The retarded susceptibility is given by

$$
\begin{aligned}
\chi(\mathbf{q}, \omega)= & -\frac{V}{\hbar} \int d \mathbf{x} \int_{0}^{\infty} d t^{\prime} e^{-i\left(\mathbf{q} \cdot \mathbf{x}-\omega t^{\prime}\right)} \\
& \times\left\langle\left[\hat{a}^{\dagger}\left(\mathbf{x}, t^{\prime}\right) \hat{a}\left(\mathbf{x}, t^{\prime}\right), \hat{a}^{\dagger}(\mathbf{0}, 0) \hat{a}(\mathbf{0}, 0)\right]\right\rangle,
\end{aligned}
$$

with $V$ the volume and $\hat{a}^{\dagger}\left(\mathbf{x}, t^{\prime}\right)$ and $\hat{a}\left(\mathbf{x}, t^{\prime}\right)$ creation and annihilation operators of the atoms. Because the atoms are in an optical lattice, we can expand the field operators in terms of the Wannier states of the lattice, which yields an expression in terms of creation and annihilation operators for every lattice site. As mentioned previously, the Hamiltonian of the system then equals the Bose-Hubbard Hamiltonian with a tunneling amplitude $t$, an on-site interaction energy $U$, and a chemical potential $\mu$. Using the decoupling approach described in Ref. [9], we can write the atomic propagator in the Mott-insulator phase as

$$
-\frac{1}{\hbar} G(\mathbf{k}, \omega)=\frac{Z(\mathbf{k})}{-\hbar \omega^{+}+\epsilon^{\mathrm{qP}}(\mathbf{k})}+\frac{1-Z(\mathbf{k})}{-\hbar \omega^{+}+\epsilon^{\mathrm{qh}}(\mathbf{k})},
$$

where the probabilities $Z(\mathbf{k})$ and $1-Z(\mathbf{k})$ account for the fact that an atomic excitation contains both quasiparticle and quasihole contributions. The notation $\hbar \omega^{+}$is shorthand for $\hbar \omega+i \xi$ with $\xi \downarrow 0$. The dispersions for the quasiparticle and quasihole excitations are given by

$$
\epsilon^{\mathrm{qp}, \mathrm{qh}}(\mathbf{k})=-\mu+\frac{U}{2}\left(2 N_{0}-1\right)+\frac{1}{2}[\epsilon(\mathbf{k}) \pm \hbar \omega(\mathbf{k})],
$$

where $N_{0}$ is the filling fraction of the lattice and the function $\epsilon(\mathbf{k})=-t \sum_{j=1}^{d} \cos 2 \pi k_{j}$ corresponds to the lattice dispersion in the experimentally relevant case of a regular square lattice. The momentum $\hbar \mathbf{k}$ is here and from now on always written in units of $2 \hbar k_{\mathrm{ph}}$, which means that the first Brillioun zone runs from $k_{j}=-1 / 2$ to $1 / 2$. The energy $\hbar \omega(\mathbf{k})$ is given by $\hbar \omega(\mathbf{k})=\sqrt{U^{2}+\left(4 N_{0}+2\right) U \epsilon(\mathbf{k})+\epsilon(\mathbf{k})^{2}}$ and the probability $Z(\mathbf{k})$ is given by $Z(\mathbf{k})=\left[U\left(2 N_{0}+1\right)+\epsilon(\mathbf{k})+\hbar \omega(\mathbf{k})\right] / 2 \hbar \omega(\mathbf{k})$.

Using the Green's function in Eq. (2), we find in general that $\chi^{0}(\mathbf{q}, \omega)=t(\mathbf{q})\left[\chi_{+}^{0}(\mathbf{q}, \omega)+\chi_{+}^{0^{*}}(-\mathbf{q},-\omega)\right]$, where $t(\mathbf{q})$ is a geometric factor that involves the appropriate overlap integral of the relevant Wannier functions [13]. Denoting integration over the first Brillouin zone as $\int_{1 \mathrm{BZ}}$, the contribution due to the creation of a particle-hole pair is given by

$$
\chi_{+}^{0}(\mathbf{q}, \omega)=\frac{1}{2} \int_{1 \mathrm{BZ}} d \mathbf{k} \frac{P(\mathbf{k}, \mathbf{k}+\mathbf{q}, \omega)}{-\hbar \omega^{+}+\epsilon^{\mathrm{qp}}(\mathbf{k}+\mathbf{q})-\epsilon^{\mathrm{qh}}(\mathbf{k})},
$$

and the time-reverse process can be written as $\chi_{-}^{0}(\mathbf{q}, \omega)$ $=\chi_{+}^{0^{*}}(-\mathbf{q},-\omega)$. Note that when evaluating the above integral, Umklapp processes have to be taken into account. This equation contains the probability $P(\mathbf{k}, \mathbf{k}+\mathbf{q}, \omega)$ for the creation of a hole with momentum $\mathbf{k}$ and a particle with momentum $\mathbf{k}$ $+\mathbf{q}$, which in a lowest-order approximation equals [1 $-Z(\mathbf{k})] Z(\mathbf{k}+\mathbf{q})$, and an energy denominator that is associated with the energy cost $\epsilon^{\mathrm{qp}}(\mathbf{k}+\mathbf{q})-\epsilon^{\mathrm{qh}}(\mathbf{k})$ of that process. This can readily be verified by taking the imaginary part of the susceptibility, which is proportional to $\int_{1 \mathrm{BZ}} d \mathbf{k} P(\mathbf{k}, \mathbf{k}$ $+\mathbf{q}, \omega) \delta\left[\hbar \omega-\epsilon^{\mathrm{qp}}(\mathbf{k}+\mathbf{q})+\epsilon^{\mathrm{qh}}(\mathbf{k})\right]$ as expected from Fermi's golden rule. The actual computation of the above integral is too complicated to do analytically, so that we have resorted to numerical methods. We have achieved this by calculating the imaginary part of Eq. (4), which roughly corresponds to integrating over the surface in the Brillouin zone where the energy denominator vanishes. In practice, this amounts to numerically finding the poles of the expression and determining their residue. The real part is calculated from the imaginary part using a Kramers-Kronig relation.

However, the results that one obtains in the lowest-order approximation do not obey particle conservation. Physically, a Raman process with momentum $\mathbf{q}$ couples to a density fluctuation $\rho(\mathbf{q})$. For zero-momentum transfer, $\rho(\mathbf{0})$ corresponds to the total number of particles and fluctuations are impossible due to particle-number conservation. If we compute the imaginary part of Eq. (4) for $\mathbf{q}=0$ we find, however, a nonzero spectrum, which means that this approach is not sufficiently accurate. The problem is due to the fact that in Eq. (2) the bare atomic propagator is not used, but a dressed propagator which contains a large self-energy correction given by

$$
\hbar \Sigma(\mathbf{k}, \omega)=2 N_{0} U-\frac{N_{0}\left(N_{0}+1\right) U^{2}}{\hbar \omega+U+\mu} .
$$

The first term on the right-hand side is the Hartree-Fock contribution, which is also present in a Bose-Einstein condensate. The second contribution is due to the correlations in the Mott insulator. Essentially this means that an atom moving through the Mott-insulating background is dressed by all the other atoms. As is known from quantum-field theory [14], one has to be careful when applying self-energy correc- 
(a)

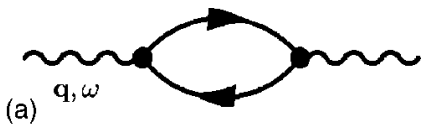

(b)

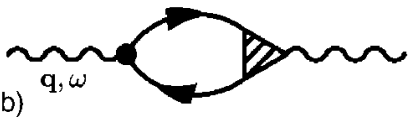

FIG. 2. Schematic representation of (a) Eq. (4) and (b) Eq. (6).

tions to the calculation of the susceptibility, because in general these corrections do not obey the required conservation laws. Using field-theoretical methods, we can derive socalled Ward identities that show that every self-energy correction requires a corresponding vertex correction in order to restore the conservation laws. Physically, this means that if the atom is dressed, we also have to dress the atom-photon coupling. Diagrammatically, this is illustrated in Fig. 2. This situation is analogous to the situation in a superconductor, where the naive BCS calculation of the electromagnetic response is not gauge invariant and a more involved approach is needed [15]. Using the relevant Ward identity [13] we find that the lowest-order probability function has to be replaced by

$$
\begin{aligned}
P(\mathbf{k}, \mathbf{k}+\mathbf{q}, \omega)= & \frac{2 \hbar \omega-\epsilon^{\mathrm{qp}}(\mathbf{k}+\mathbf{q})+\epsilon^{\mathrm{qh}}(\mathbf{k})}{\hbar \omega(\mathbf{k}+\mathbf{q})+\hbar \omega(\mathbf{k})} \\
& \times[Z(\mathbf{k}+\mathbf{q})-Z(\mathbf{k})] .
\end{aligned}
$$

Note that the probability now vanishes when $\mathbf{q} \rightarrow 0$, so that particle conservation is indeed no longer violated. In fact, for small $\mathbf{q}$ and $\hbar \omega$ just above threshold $P \propto q^{2} / \Delta_{0}^{2}$, where $\Delta_{0}$ is the gap for particle-hole excitations.

\section{RESULTS}

In Figs. 3 and 4 the result of a numerical integration of Eqs. (4) and (6) is shown in two and three dimensions, respectively. Both calculations have been carried out for a

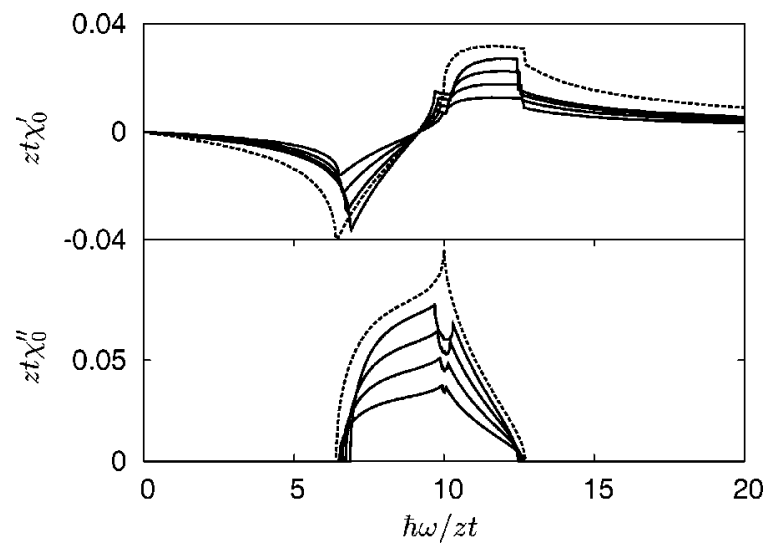

FIG. 3. Real $\left(\chi_{0}^{\prime}\right)$ and imaginary $\left(\chi_{0}^{\prime \prime}\right)$ parts of the susceptibility for $U / z t=10$ and $\mathbf{q}=0.10,0.14,0.18,0.20$ along a lattice direction, in two dimensions. The dotted line in the bottom figure is the result for $\mathbf{q}=0.001$ multiplied by 250 to show the behavior for small $q$.

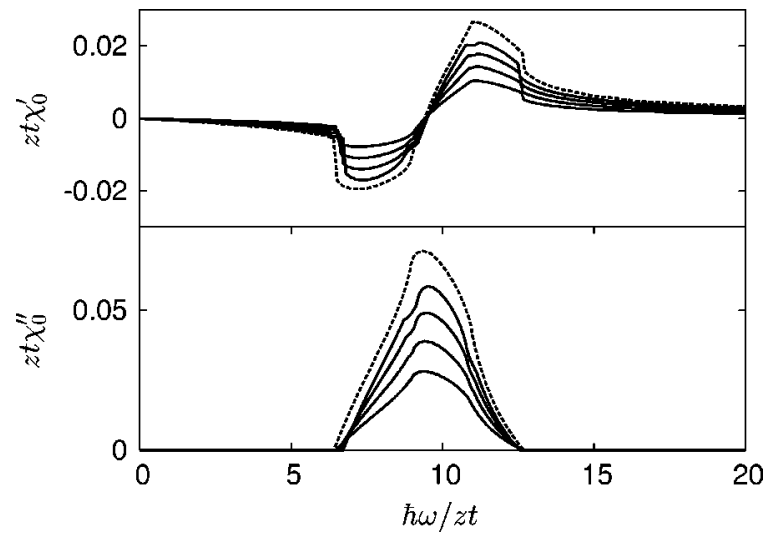

FIG. 4. Real and imaginary parts of the susceptibility for $U / z t$ $=10$ and $\mathbf{q}=0.10,0.14,0.18,0.20$ along a lattice direction, in three dimensions. The dotted line in the bottom figure is the result for $\mathbf{q}=0.001$ multiplied by 250 to show the behavior for small $q$.

regular square lattice and the momentum $\mathbf{q}$ is chosen along a principal lattice direction. All energies in the following figures are given in units $z t$, where $z$ is the coordination number of the lattice.

The imaginary part of Fig. 3 clearly shows singularities around $\hbar \omega=U$. These singularities are due to the fact that there are saddle points in the dispersion and that a saddle point in the dispersion causes an integrable singularity in the density of states. These are so-called van Hove singularities [16]. It is interesting to see that the van Hove singularities split up as the momentum is increased, which is caused by the fact that the saddle-point energy in the direction of $\mathbf{q}$ and the saddle-point energy in the orthogonal direction(s) are shifted by different amounts. This is also visible in Fig. 4. However, it is less clear in this case, because the van Hove singularities are more smeared out in three dimensions. Also, the opening of the threshold for the two-photon absorption in the three-dimensional case is far less steep than in the twodimensional case. Note that when additional harmonic confinement is present, some background signal is expected due to the superfluid shell around the Mott insulator. To investigate possible collective modes in this system, we determined higher-order corrections in the random-phase approximation (RPA). It can be shown that in RPA the susceptibility is given by $\chi(\mathbf{q}, \omega)=\chi^{0}(\mathbf{q}, \omega) /\left[1-2 U \chi^{0}(\mathbf{q}, \omega)\right]$. This means that there is a resonance in the scattering rate when the real part of $\chi^{0}(\mathbf{q}, \omega)$ is equal to $1 / 2 U$. However, as can be seen from Figs. 3 and 4, the real parts in both cases are rather small compared to $1 / U$ for experimentally relevant values of $U$, which are typical in the order of $U / z t \approx 10$ [3]. Therefore, including the RPA denominator does not qualitatively change our previous results. Of course, for much larger values of $U$ the RPA denominator always becomes important.

In Fig. 5 we plot the imaginary part of $\chi_{0}$, for a range of values for the coupling constant $U / z t$, and for a fixed momentum $\mathbf{q}=0.10$. We see that the threshold behavior becomes steeper as we approach the critical value of $U_{c} / z t$ $\approx 5.83$. This steepening is caused by the fact that close to the quantum critical point, the dispersion stiffens and becomes almost linear, as illustrated in Fig. 1(b). We also see that 


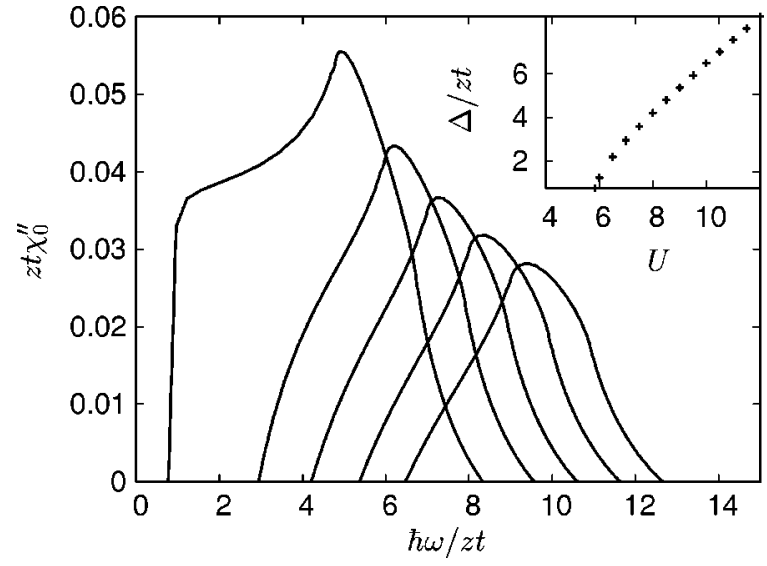

FIG. 5. Imaginary part of the susceptibility in a threedimensional lattice for $\mathbf{q}=0.10$ along a lattice direction and $U / z t$ $=5.83,7,8,9,10$.

there remains a nonzero gap when $U=U_{c}$. This is due to the fact that we are not considering a zero-momentum excitation, due to the reasons given above. In the inset of Fig. 5, we plot this gap $\Delta_{q}$ as a function of $U / z t$. For large $U$ the gap grows linearly with $U$ and for $U$ close to $U_{c}$, the gap closes more rapidly. In the case of $\mathbf{q}=\mathbf{0}$ the gap would in our mean-field approximation close as $\sqrt{U-U_{c}}$ when $U \downarrow U_{c}$, but for small nonzero $\mathbf{q}$ it closes as $\sqrt{U-U_{c}+\eta q^{4}}$, where the factor $\eta$ is a positive function of $U_{c}$ and $t$.

\section{DISCUSSION}

In summary, we have proposed a means of studying Mott insulators in optical lattices, using the relatively well-known technique of Bragg spectroscopy. We have presented spectra that can be measured directly by trap loss or heating measurements. In a recent experiment by Stöferle et al. [17] the authors use a setup where the laser beams are perfectly counterpropagating, which corresponds to a quasimomentum transfer of zero. As we have argued above, there should be no scattering in that case and the signal can only be due to nonlinear response or due to the fact that the system is inhomogeneous and of finite size. We have found that by measuring the threshold behavior of the two-photon scattering rate at various quasimomenta, it is possible to determine the gap by extrapolation. We have shown that for a theoretical description of Bragg spectroscopy on the Mott insulator it is absolutely essential to dress the photon-atom coupling. As a result it turns out that although it is common to use the language of solid-state physics to describe these systems, the physics is quantitatively, and even qualitatively, very different due to the many-body effects.
[1] L.-M. Duan, E. Demler, and M. D. Lukin, Phys. Rev. Lett. 91, 090402 (2003).

[2] L. Santos, M. A. Baranov, J. I. Cirac, H.-U. Everts, H. Fehrmann, and M. Lewenstein, Phys. Rev. Lett. 94, 030601 (2004).

[3] M. Greiner, O. Mandel, T. Esslinger, T. W. Hänsch, and I. Bloch, Nature (London) 415, 39 (2002).

[4] M. P. A. Fisher, P. B. Weichman, G. Grinstein, and D. S. Fisher, Phys. Rev. B 40, 546 (1989).

[5] D. Jaksch, C. Bruder, J. I. Cirac, C. W. Gardiner, and P. Zoller, Phys. Rev. Lett. 81, 3108 (1998).

[6] G. K. Brennen, C. M. Caves, P. S. Jessen, and I. H. Deutsch, Phys. Rev. Lett. 82, 1060 (1999).

[7] E. Roddick and D. Stroud, Phys. Rev. B 48, 16600 (1993).

[8] K. Sheshadri, H. R. Krishnamurthy, R. Pandit, and T. V. Ramakrishnan, Europhys. Lett. 22, 257 (1993).

[9] D. van Oosten, P. van der Straten, and H. T. C. Stoof, Phys.
Rev. A 63, 053601 (2001).

[10] M. Kozuma, L. Deng, E. W. Hagley, J. Wen, R. Lutwak, K. Helmerson, S. L. Rolston, and W. D. Phillips, Phys. Rev. Lett. 82, 871 (1998).

[11] D. M. Stamper-Kurn, A. P. Chikkatur, A. Gorlitz, S. Inouye, S. Gupta, D. E. Pritchard, and W. Ketterle, Phys. Rev. Lett. 83, 2876 (1999).

[12] G. Grynberg and C. Robiliard, Phys. Rep. 355, 335 (2001).

[13] D. van Oosten et al. (unpublished).

[14] J. Zinn-Justin, Quantum Field Theory and Critical Phenomena (Oxford, New York, 1989).

[15] J. R. Schrieffer, Theory of Superconductivity (Benjamin, New York, 1964).

[16] L. van Hove, Phys. Rev. 89, 1189 (1953).

[17] T. Stöferle, H. Moritz, C. Schori, M. Köhl, and T. Esslinger, Phys. Rev. Lett. 92, 130403 (2004). 